\title{
Iron Chelator or Iron Supplement Consumption in COVID-19? The Role of Iron with Severity Infection
}

\author{
Alireza Bastin ${ }^{1} \cdot$ Hamidreza Shiri ${ }^{1} \cdot$ Sareh Zanganeh $^{2} \cdot$ Saba Fooladi $^{3} \cdot$ Mohammad Amin Momeni Moghaddam $^{4}$. \\ Mehrnaz Mehrabani ${ }^{5} \cdot$ Mohammad Hadi Nematollahi $^{1}$ (1)
}

Received: 5 October 2021 / Accepted: 22 November 2021 / Published online: 25 November 2021

(c) The Author(s), under exclusive licence to Springer Science+Business Media, LLC, part of Springer Nature 2021

\begin{abstract}
Iron is a trace element that is used to replicate the virus and has a role in the vital functions of the body and the host's innate immune system. The mechanism of iron in COVID-19 severity is still not well understood. The aim of this study was to evaluate the association of the iron with COVID-19 severity. A case-control study was performed on 147 patients with a positive PCR test result and 39 normal individuals admitted to the Persian Gulf Martyrs Hospital in Bushehr, Iran. The iron profiles and related tests were measured along with hematological analytes. Hemoglobin ( $\mathrm{Hb}), \mathrm{Fe}$, and saturated transferrin decreased in all the groups compared to the controls, but ferritin increased in the patient groups. After adjusting for age and sex, we found that increased ferritin levels augmented the odds ratio $(\mathrm{OR})$ of the disease in the moderate $(\mathrm{OR}=2.95$, $P=0.007)$, severe $(\mathrm{OR}=6.1, P<0.001)$, and critical groups $(\mathrm{OR}=8.34, P<0.001)$. The decreased levels of Fe reduced the $\mathrm{OR}$ of the disease in the mild $(\mathrm{OR}=0.96, P<0.001)$, moderate $(\mathrm{OR}=0.96, P<0.001)$, severe $(\mathrm{OR}=0.95, P<0.001)$, and critical $(\mathrm{OR}=0.98, P=0.001)$ groups. $\mathrm{Fe}(\mathrm{AUC}=85.95$, cutoff $<75.5 \mu \mathrm{g} / \mathrm{dL}, P<0.001)$ and ferritin $(\mathrm{AUC}=84.45$, cutoff $>157.5 \mathrm{ng} / \mathrm{dL}, P<0.001$ ) have higher AUC for disease prognosis, but only ferritin (AUC $=74.89$, cutoff $>261.5 \mathrm{ng} /$ $\mathrm{dL}, P<0.001)$ has higher AUC for disease severity assays. It could be concluded that the use of iron chelators to reduce iron intake can be considered a therapeutic goal. In addition, measuring Fe and ferritin is beneficial for the diagnosis of the disease and determining its severity.
\end{abstract}

Keywords COVID- $19 \cdot \mathrm{Fe} \cdot$ Ferritin $\cdot$ Disease severity $\cdot$ Iron chelator

Alireza Bastin and Hamidreza Shiri contributed equally to this work.

Mohammad Hadi Nematollahi

mh.nematollahi@yahoo.com; m.nematollahi@kmu.ac.ir

1 Student Research Committee, Kerman University of Medical Sciences, Kerman, Iran

2 Bacteriology \& Virology Department, Shiraz Medical School, Shiraz University of Medical Sciences, Shiraz, Iran

3 Department of Clinical Biochemistry, Afzalipoor Faculty of Medicine, Kerman University of Medical Sciences, Kerman, Iran

4 Department of Nutrition and Biochemistry, Faculty of Medicine, Infectious Diseases Research Center, Gonabad University of Medical Sciences, Gonabad, Iran

5 Physiology Research Center, Institute of Neuropharmacology, Kerman University of Medical Sciences, Kerman, Iran

\section{Introduction}

In 2003, coronaviruses (CoVs) caused severe acute respiratory syndrome (SARS) in China. In late 2019, Chinese researchers identified a novel CoVs called SARS-CoV-2 in Wuhan which causes the coronavirus disease (COVID-19) [1]. Due to the rapid transmission and spread of the disease, the World Health Organization (WHO) reported COVID19 as a global health concern [2, 3]. Hypoxia, as a clinical symptom, may play a role in the prognosis of COVID-19 because most of the patients who require intensive care have an atypical form of acute respiratory distress syndrome (ARDS) with preserved lung gas [4]. Because of its heme ring and the iron in its structure, hemoglobin $(\mathrm{Hb})$ is able to transfer oxygen from the lungs to various tissues [5]. Therefore, a disorder in the iron metabolism and hemoglobinopathy may remarkably compromise the capacity of red blood cells (RBCs) to transport $\mathrm{O}_{2}$ [6]. 
Iron is an essential nutrient for vital activities in eukaryotic and prokaryotic cells [7]. Many proteins, $\mathrm{Hb}$, and enzymes involved in the DNA and RNA functions, such as ribonucleotide reductase and DNA primase, have irondependent activities [8]. In addition, several indicators were also proposed for the evaluation of iron status, including $\mathrm{Hb}$, mean cell volume (MCV), mean cell hemoglobin $(\mathrm{MCH})$, serum ferritin, soluble transferrin receptors (sTfR), transferrin saturation, and total iron-binding capacity (TIBC) [9]. Transferrin, as the main carrier of iron, TIBC represents the maximum amount of iron that can bind to transferrin, and saturated transferrin is obtained via iron/TIBC $\times 100$ [10].

Viral performance relies on the host cell, and it must contain high amounts of iron for replication and protein synthesis. Iron deficiency leads to the cessation of the activity of iron-dependent proteins and impairs the physiological functions of cells [11]. Studies have shown that iron regulation is involved in the host cell defense mechanism, where deficiency of iron leads to a relative resistance against viral infections [12], and the increase of iron levels is associated with the expansion of the virus population [8]. So far, many studies have been conducted on the effect of iron levels on the onset of symptoms and treatment of viral diseases, HBV, HCV, and HIV [13]. For example, it has been reported that high levels of iron reduce the viability of HIV-infected cells [14], and the progression of HCV infection is inhibited by the administration of exogenous iron $[15,16]$. Moreover, several studies have reported that serum iron levels are decreased in patients with COVID-19 [17]. Therefore, the relationship of the iron profile with COVID-19 may be different from its relationship with other viral infections.

Considering the important role of iron in the determination of prognosis and improvement in the treatment of viral infections, and because alterations in the blood iron profile and its regulatory mechanism in COVID-19 patients are not well known, the purpose of the present study was to investigate the association of the iron profile in COVID-19 patients with the severity of the clinical symptoms. The results can be beneficial in the determination of prognosis, treatment procedures, and choosing an effective medication.

\section{Material and Methods}

\section{Subjects}

As a cross-sectional study, the present research was carried out on a total of 147 COVID-19 patients and 39 normal individuals admitted to the Persian Gulf Martyrs Hospital of Bushehr University of Medical Sciences, Bushehr, Iran, from May 2020 to September 2020 (DMS (degrees $\left(^{\circ}\right)$, minutes $(')$, seconds (")) latitude and longitude coordinates for the hospital are $\left.28^{\circ} 56^{\prime} 16.7^{\prime \prime} \mathrm{N} 50^{\circ} 52^{\prime} 04.1^{\prime \prime} \mathrm{E}\right)$. The inclusion criteria were as follows: patients aged $\geq 20$ years admitted to the hospital with symptoms suggestive of COVID-19, who had a positive result for the real-time PCR test of COVID-19 and had signed an informed consent form. Exclusion criteria included consumption of drugs such as iron chelators and anemia tablets, allergy to any medication, severe liver disease, renal insufficiency (GFR $<60 \mathrm{~mL} / \mathrm{min}$ ), or on any form of renal replacement therapy, pregnancy or breastfeeding, anticipated discharge from the hospital, or scheduled transfer to another hospital that was not a study site within $48 \mathrm{~h}$. The selected patients were divided into four groups according to clinical manifestations, including (1) patients with mild COVID-19 infection who had symptoms such as fever, cough, or change in sense of taste or smell with no dyspnea ( $n=33)$, (2) patients with moderate COVID-19 infection and symptoms such as clinical or radiographic evidence of lower respiratory tract disease and oxygen saturation $\geq 94$ $(n=37)$, (3) patients with severe COVID-19 infection and symptoms such as oxygen saturation $<94 \%$, respiratory rate $\geq 30$ breaths/min, and lung infiltrates $>50 \%(n=34)$, and (4) patients with critical COVID-19 infection and symptoms such as respiratory failure, shock, and multi-organ dysfunction or failure $(n=43)$, and the control group $(n=39)$, which was selected according to the study by Rajesh et al. [18]. This study abides by the Declaration of Helsinki on research involving human subjects and is approved by the Ethics Committee of Kerman University of Medical Sciences, Kerman, Iran (IR.KMU.REC.1399.239).

\section{Samples and Data Collection}

Demographic information was recorded through interviews with the participants. About $10 \mathrm{~mL}$ of venous blood samples were drawn from each patient and poured into two separate tubes, one containing EDTA for routine hematology tests and the other containing a gel separator for evaluating serological and biochemical parameters. For the measurement of serological and biochemical analytes, the blood samples were centrifuged ( $8 \mathrm{~min}$ at $2500 \mathrm{rpm}$ ) and serum was separated and stored at $-20^{\circ} \mathrm{C}$.

\section{Measurement of Biochemical Analytes}

The serum levels of iron (Fe), TIBC, transferrin (Tf), and high-sensitivity C-reactive protein (hs-CRP) were determined by means of the standard kits (Pars Azmoon, Tehran, Iran) using an autoanalyzer (Selectra XL instrument; Randox Laboratories Ltd, Antrim, UK) in a standard laboratory context [19]. Ferritin serum level was evaluated by enzyme-linked immunosorbent assay (ELISA) (Monobind, CA, USA). 


\section{Measurement of Hematological Analytes}

Total leukocyte count, differential leukocyte count, RBC count, $\mathrm{Hb}$ concentration, total platelet count (PLT), hematocrit value (Hct), and blood indices (MCHC, $\mathrm{MCH}$, and $\mathrm{MCV}$ ) were evaluated via an automated hematological analyzer (Sysmex). Also, ESR was assessed by an automated ESR analyzer (LENA, SPAIN).

\section{Statistical Analysis}

All continuous variables were represented as mean \pm standard errors of the mean (SEM), while categorical variables were represented by numbers (\%). The Shapiro-Wilks test was used to determine data distribution. To demonstrate the differences between the groups, one-way analysis of variance (ANOVA)/Kruskal-Wallis with post hoc Tukey/ Mann-Whitney $U$ tests and chi-square test were employed. Spearman's rho correlation coefficient was used to demonstrate the relationships between continuous variables. Linear regression was applied to determine the effects of continuous parameters (as independent variables) on the iron profile element (as a dependent variable). Due to the skewed distributions of the frequency levels of ferritin, they were $\mathrm{ln}$ converted to enhance the linear model's normality assumption. The associations between disease severity (mild, moderate, severe, and critical) and the iron profile were estimated using the continuous logistic regression model, based on adjustments for age and gender. The built interaction model was assessed using receiver operating characteristic (ROC) analysis and area under the curve (AUC) with 95\%
CI calculations. The ROC curves for models predicted the impact of each iron profile element on COVID-19 development in patients vs control and mild vs severe and critical groups. Cutoff values were computed for each of the biomarkers with the highest sensitivity and specificity. $P$-values $<0.05$ were considered statistically significant. SPSS software version 22.0 for windows (SPSS Inc., Chicago, IL) was used for the statistical analyses, and charts were plotted using GraphPad Prism software (8.4.3).

\section{Results}

\section{Demographic Characteristics of the Study Participants}

According to Table S1, the mild group included 33 (17\%) patients $(19$ male $(18.1 \%))$, the moderate group consisted of $37(19.9 \%)$ patients (19 male (18.1\%)), the severe group included 34 (18.3\%) patients (17 male (16.2\%)), the critical group had $43(21.3 \%)$ patients (32 male $(30.5 \%)$ ), and the normal group included 39 (21\%, 18 male (17.1\%)). No statistical difference was observed between the groups in terms of the number of patients $(p=0.081)$.

\section{Iron Profile}

The iron profile is shown in Fig. 1 and the female and male categories are represented in Table S1. Fe and transferrin saturation levels decreased in all the patient groups when compared to control $(P<0.001)$. Ferritin increased in the
Fig. 1 Comparison of iron profile in case and control groups. (a) Fe, (b) TIBC, (c) transferrin, (d) ferritin, (e) transferrin saturation, in the mild, moderate, severe, and critical groups. Parameters are presented as mean \pm SEM and significance level is as: $* P<0.05$, $* * P<0.01$, *** $P<0.001$
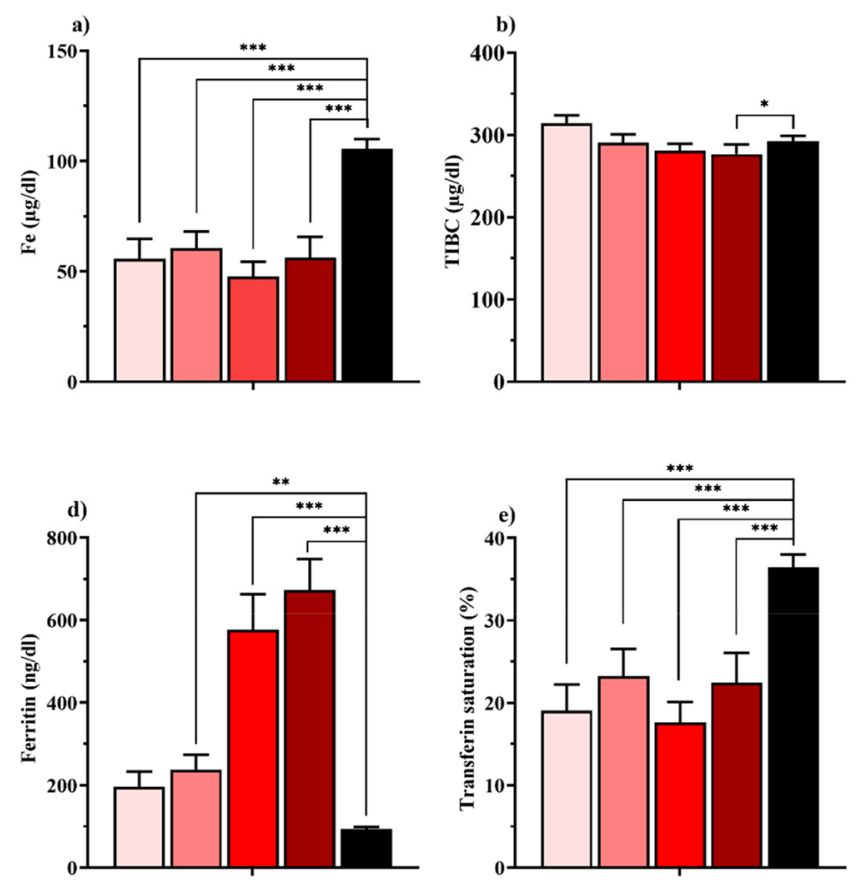

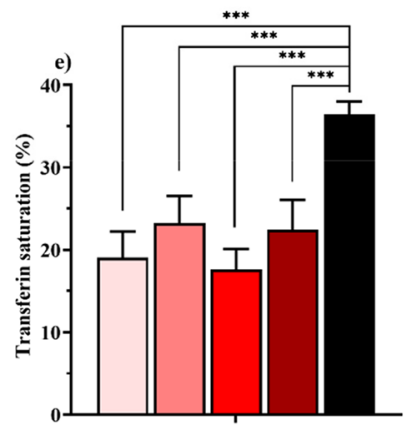

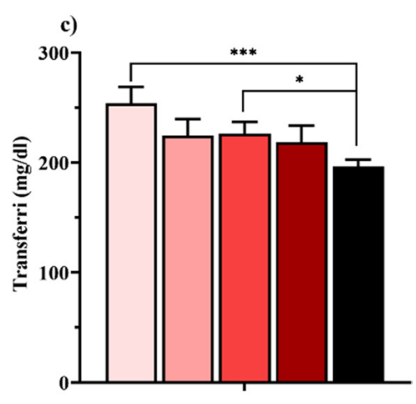

Mild

Moderate

Severe

Critical

Normal 
moderate $(p=0.006)$, severe, and critical $(p<0.001)$ groups compared to the control. TIBC was only lower in the critical group than the normal $(p=0.048)$. Transferrin increased in the mild $(p<0.001)$ and severe $(p=0.012)$ groups compared to the normal group.

\section{Hematological Index}

The hematological profile is shown in Fig. 2 and the female and male categories are represented in Table S2. White blood cell (WBC) count was lower in the mild group than the normal $(P=0.001)$. Hb, MCV, and $\mathrm{MCH}$ decreased in all the groups compared to the normal group $(P<0.001)$. HCT in the mild $(P=0.001)$, moderate, severe, and critical groups $(P<0.001)$ was lower than in the control group. MCHC was higher in the normal group than the moderate $(P=0.046)$, severe $(P=0.001)$, and critical $(P=0.025)$ groups. PLT decreased in the mild $(P<0.001)$ and moderate $(P=0.027)$ groups compared to the normal. Red blood cell distribution width (RDW) increased all the groups when compared to the normal group $(P<0.001)$. The $\mathrm{N} / \mathrm{L}$ ratio, ESR, and hsCRP were higher in all the groups than the normal group $(P<0.001)$.

\section{Correlation Coefficient}

Table 1 depicts the relationship between the iron profile and the hematological indices along with the Spearman rank correlation test results. The Spearman rank correlation coefficient values varied from 0.972 to -0.846 . The results revealed that $\mathrm{Fe}$ had a positive correlation with $\mathrm{Hb}, \mathrm{HCT}$, $\mathrm{MCV}$, and $\mathrm{MCH}$ and a negative association with $\mathrm{N} / \mathrm{L}$ ratio,
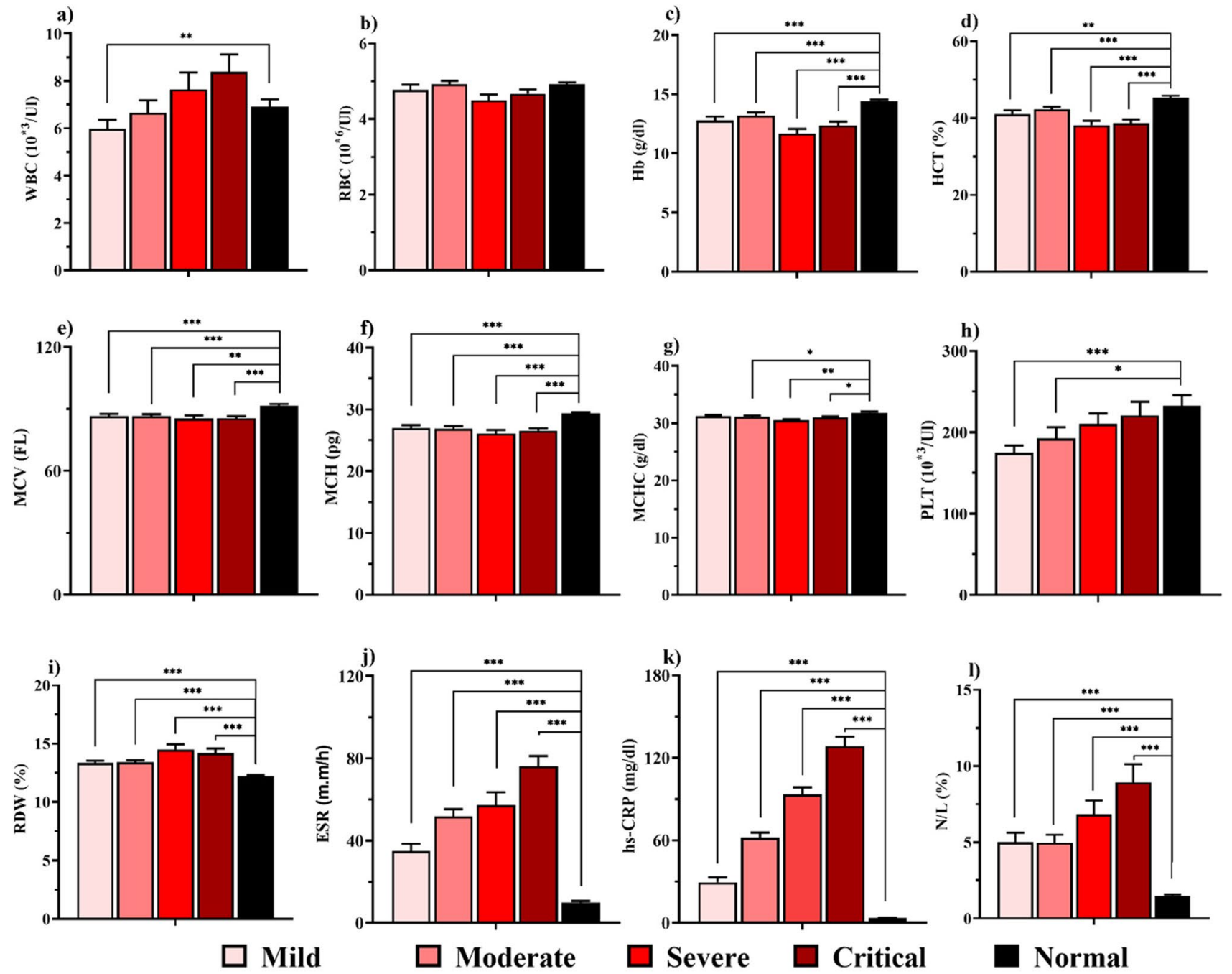

Fig. 2 Hematological index in the mild, moderate, severe, and critical groups. Parameters are presented as mean \pm SEM; (a) WBC, (b) $\mathrm{RBC},(\mathbf{c}) \mathrm{Hb},(\mathbf{d}) \mathrm{HCT},(\mathbf{e}) \mathrm{MCV}$, (f) $\mathrm{MCH},(\mathbf{g}) \mathrm{MCHC}$, (h) PLT, (i)
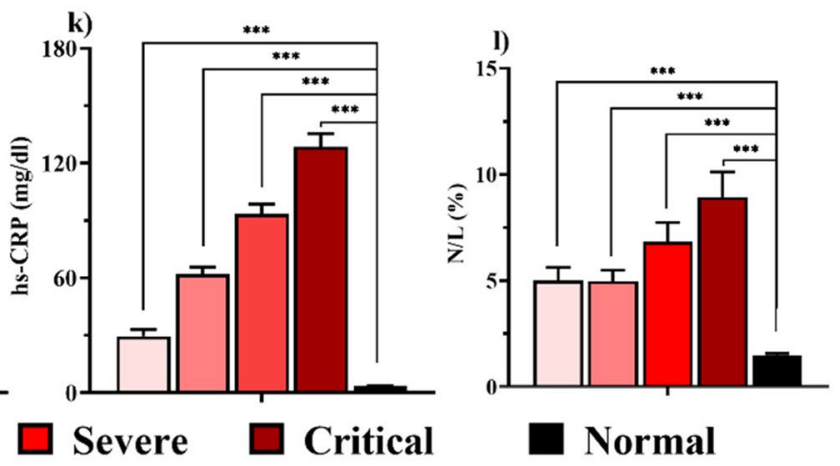

RDW, (j) ESR, (k) hs-CRP, (l) N/L ratio. Significance level is as: $* P<0.05, * * P<0.01, * * * P<0.001$ 
Table 1 Spearman's correlation of the iron profile with the hematological index

\begin{tabular}{lccllc}
\hline Variable & Fe & Transferrin saturation & Transferrin & TIBC & Ferritin \\
\hline WBC & 0.111 & $0.169^{*}$ & $-0.221^{* *}$ & $-0.251^{* *}$ & 0.062 \\
RBC & -0.012 & -0.026 & 0.066 & 0.092 & $-0.152^{*}$ \\
Hb & $0.183^{*}$ & $0.147^{*}$ & -0.036 & 0.067 & $-0.186^{*}$ \\
$\mathrm{HCT}$ & $0.161^{*}$ & 0.128 & -0.030 & 0.064 & $-0.216^{* *}$ \\
MCV & $0.225^{* *}$ & $0.199^{* *}$ & -0.123 & -0.038 & -0.016 \\
MCH & $0.267^{* *}$ & $0.234^{* *}$ & -0.125 & 0.000 & -0.055 \\
N/L ratio & $-0.320^{* *}$ & $-0.240^{* *}$ & -0.007 & $-0.222^{* *}$ & $0.377^{* *}$ \\
ESR & $-0.364^{* *}$ & $-0.297^{* *}$ & 0.055 & $-0.187^{*}$ & $0.312^{* *}$ \\
hs-CRP & $-0.392^{* *}$ & $-0.299^{* *}$ & -0.004 & $-0.244^{* *}$ & $0.516^{* *}$ \\
Fe & 1.000 & $0.974^{* *}$ & $-0.722^{* *}$ & $-0.286^{* *}$ & $-0.148^{*}$ \\
Transferrin saturation & & 1.000 & $-0.846^{* *}$ & $-0.468^{* *}$ & -0.080 \\
Transferrin & & & 1.000 & $0.798^{* *}$ & -0.143 \\
TIBC & & & 1.000 & $-0.242^{* *}$ \\
Ferritin & & & & 1.000 \\
\hline
\end{tabular}

**Correlation is significant at the 0.01 level (2-tailed)

*Correlation is significant at the 0.05 level (2-tailed)
ESR, and hs-CRP. Transferrin saturation had a positive correlation with $\mathrm{WBC}, \mathrm{Hb}, \mathrm{MCV}, \mathrm{MCH}$, and $\mathrm{Fe}$ and an inverse association with N/L ratio, ESR, and hs-CRP. Transferrin had a negative correlation with WBC, Fe, and transferrin saturation. TIBC had a positive correlation with transferrin and a negative correlation with $\mathrm{WBC}, \mathrm{N} / \mathrm{L}$ ratio, ESR, hs-CRP, Fe, and transferrin saturation. Ferritin had a direct correlation with $\mathrm{N} / \mathrm{L}$ ratio, ESR, and hs-CRP and inverse correlation with RBC, Hb, HCT, Fe, and TIBC.

\section{Linear Regression Analysis}

A linear regression analysis was conducted to evaluate the impact of the hematological markers on the development of iron profile element and the results are shown in Table 2. Fe was positively associated with $\mathrm{MCV}, \mathrm{MCH}$, and had a negative relationship with N/L ratio, ESR, and hs-CRP. Transferrin saturation was negatively associated with ESR and hs-CRP. TIBC negatively associated with WBC, N/L ratio, ESR, and hs-CRP. In-ferritin was positively associated with, $\mathrm{N} / \mathrm{L}$ ratio, ESR, and hs-CRP and negatively associated with $\mathrm{RBC}, \mathrm{Hb}$, and HCT.

\section{Logistic Regression}

Logistic regression was performed to predict the effect of the iron profile on disease severity in mild, moderate, severe, and critical groups, and the results are shown in Table 3 along with the results adjusted for age and gender. According to these results, ln-ferritin was associated with disease severity in moderate $(\mathrm{OR}=2.95, P=0.007)$, severe $(\mathrm{OR}=6.1, P<0.001)$, and critical $(\mathrm{OR}=8.34$, $P<0.001)$ groups. Fe was found to be inversely related to disease severity in mild $(\mathrm{OR}=0.967, P<0.001)$, moderate (OR $=0.969, P<0.001)$, severe $(\mathrm{OR}=0.956, P<0.001)$, and critical $(\mathrm{OR}=0.981, P=0.001)$ cases. Transferrin saturation was also inversely associated with disease severity in mild (OR $=0.914, P<0.001)$, moderate $(\mathrm{OR}=0.944, P=0.001)$, severe $(\mathrm{OR}=0.892, P<0.001)$, and critical $(\mathrm{OR}=0.964$, $P=0.012$ ) groups. Both $\mathrm{Fe}$ and transferrin saturation were shown to be inversely related to the severity of COVID-19. Transferrin association with COVID-19 severity in the mild $(\mathrm{OR}=1.015, P=0.002)$ and severe $(\mathrm{OR}=1.013, P=0.016)$.

\section{ROC Curve}

ROC analysis of iron parameters for disease prognosis (patients vs control) and disease severity (mild vs severe and critical) and the best cutoff method performed and the results are shown in Fig. 3 and Table S3. The iron parameters for disease prognosis and disease severity with the highest AUC were in the following order respectively: $\mathrm{Fe}$, ferritin, $\mathrm{Hb}$, and ferritin.

For disease prognosis, Fe had an AUC of 85.95 (cutoff $<75.5 \mathrm{mg} / \mathrm{dL}, P<0.001)$ and this for disease severity is not significant $(P=0.43)$. Ferritin had an AUC of 84.45 for disease prognosis (cutoff $>157.5 \mathrm{ng} / \mathrm{dL}, P<0.001$ ), and the best parameter for disease severity had an AUC of 72.89 (cutoff $>261 \mathrm{ng} / \mathrm{dL}, P<0.001$ ). Transferrin had an AUC of 64.43 (cutoff $>238 \mathrm{mg} / \mathrm{dL}, P=0.005$ ) for disease prognosis and this for disease severity had an AUC of 62.12 (cutoff $>242.5 \mathrm{mg} / \mathrm{dL}, P=0.044$ ). Hb had an AUC of 81.71 (cutoff $<13.35 \mathrm{~g} / \mathrm{dL}, P<0.001$ ) for disease prognosis and had an AUC of 62.86 (cutoff $<10.75 \mathrm{~g} / \mathrm{dL}, P=0.03$ ) for disease severity. Also, TIBC was not significant for disease 


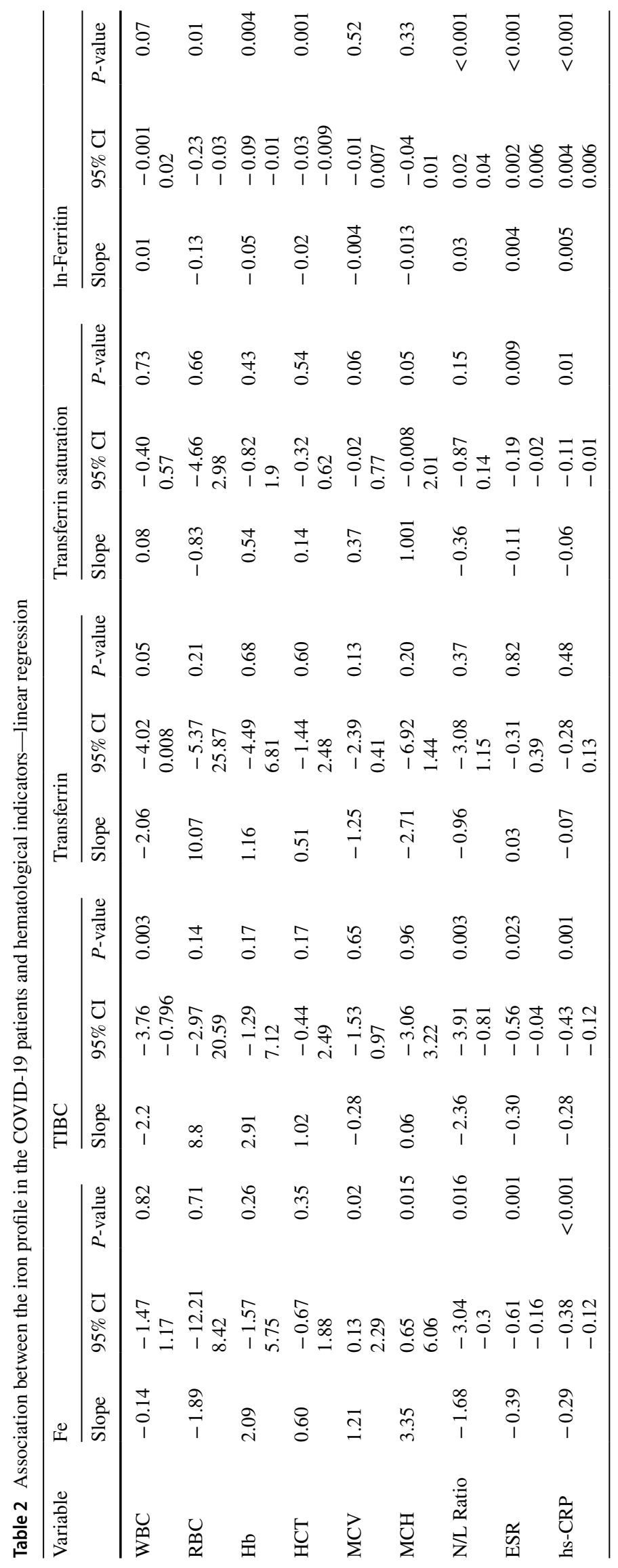


Table 3 Association between COVID-19 incidence and iron profile-logistic regression (adjusted for age and gender)

\begin{tabular}{|c|c|c|c|c|c|c|c|c|c|}
\hline \multirow[t]{2}{*}{ Variable } & \multirow[t]{2}{*}{ Groups } & \multicolumn{4}{|l|}{ Crude } & \multicolumn{4}{|c|}{ Adjusted } \\
\hline & & $B$ & OR & $95 \%$ CI & $P$-value & $B$ & OR & $95 \%$ CI & $P$-value \\
\hline \multirow[t]{4}{*}{ ln-Ferritin } & Mild & 0.257 & 1.29 & $\begin{array}{l}0.745 \\
2.34\end{array}$ & 0.362 & 0.243 & 1.25 & $\begin{array}{l}0.724 \\
2.24\end{array}$ & 0.4 \\
\hline & Moderate & 1.17 & 3.25 & $\begin{array}{l}1.53 \\
6.89\end{array}$ & 0.002 & 1.08 & 2.95 & $\begin{array}{l}1.33 \\
6.51\end{array}$ & 0.007 \\
\hline & Severe & 2.036 & 7.65 & $\begin{array}{l}2.69 \\
19.74\end{array}$ & $<0.001$ & 1.81 & 6.1 & $\begin{array}{l}2.24 \\
16.64\end{array}$ & $<0.001$ \\
\hline & Critical & 2.17 & 8.77 & $\begin{array}{l}3.31 \\
23.23\end{array}$ & $<0.001$ & 2.12 & 8.34 & $\begin{array}{l}3.11 \\
22.39\end{array}$ & $<0.001$ \\
\hline \multirow[t]{4}{*}{$\mathrm{Fe}$} & Mild & -0.033 & 0.967 & $\begin{array}{l}0.952 \\
0.983\end{array}$ & $<0.001$ & -0.033 & 0.967 & $\begin{array}{l}0.952 \\
0.983\end{array}$ & $<0.001$ \\
\hline & Moderate & -0.03 & 0.97 & $\begin{array}{l}0.956 \\
0.985\end{array}$ & $<0.001$ & -0.031 & 0.969 & $\begin{array}{l}0.955 \\
0.984\end{array}$ & $<0.001$ \\
\hline & Severe & -0.047 & 0.954 & $\begin{array}{l}0.935 \\
0.973\end{array}$ & $<0.001$ & -0.045 & 0.956 & $\begin{array}{l}0.937 \\
0.976\end{array}$ & $<0.001$ \\
\hline & Critical & -0.022 & 0.979 & $\begin{array}{l}0.976 \\
0.99\end{array}$ & $<0.001$ & -0.019 & 0.981 & $\begin{array}{l}0.97 \\
0.992\end{array}$ & 0.001 \\
\hline \multirow[t]{4}{*}{ TIBC } & Mild & 0.01 & 1.01 & $\begin{array}{l}0.999 \\
1.02\end{array}$ & 0.067 & 0.009 & 1.01 & $\begin{array}{l}0.999 \\
1.02\end{array}$ & 0.071 \\
\hline & Moderate & -0.001 & 0.999 & $\begin{array}{l}0.999 \\
1.008\end{array}$ & 0.856 & 0.002 & 1.002 & $\begin{array}{l}0.993 \\
1.01\end{array}$ & 0.697 \\
\hline & Severe & -0.006 & 0.994 & $\begin{array}{l}0.983 \\
1.005\end{array}$ & 0.26 & -0.002 & 0.998 & $\begin{array}{l}0.986 \\
1.01\end{array}$ & 0.727 \\
\hline & Critical & -0.004 & 0.996 & $\begin{array}{l}0.989 \\
1.003\end{array}$ & 0.25 & -0.004 & 0.996 & $\begin{array}{l}0.989 \\
1.004\end{array}$ & 0.341 \\
\hline \multirow[t]{4}{*}{ Transferrin } & Mild & 0.015 & 1.015 & $\begin{array}{l}1.006 \\
1.025\end{array}$ & 0.001 & 0.015 & 1.015 & $\begin{array}{l}1.006 \\
1.024\end{array}$ & 0.002 \\
\hline & Moderate & 0.006 & 1.006 & $\begin{array}{l}0.999 \\
1.013\end{array}$ & 0.084 & 0.008 & 1.008 & $\begin{array}{l}1.001 \\
1.015\end{array}$ & 0.31 \\
\hline & Severe & 0.011 & 1.011 & $\begin{array}{l}1.002 \\
10.21\end{array}$ & 0.021 & 0.013 & 1.013 & $\begin{array}{l}1.003 \\
1.024\end{array}$ & 0.016 \\
\hline & Critical & 0.004 & 1.004 & $\begin{array}{l}0.998 \\
1.01\end{array}$ & 0.184 & 0.004 & 1.004 & $\begin{array}{l}0.998 \\
1.01\end{array}$ & 0.216 \\
\hline \multirow[t]{4}{*}{ Transferrin saturation } & Mild & -0.089 & 0.914 & $\begin{array}{l}0.875 \\
0.976\end{array}$ & $<0.001$ & -0.089 & 0.914 & $\begin{array}{l}0.876 \\
0.956\end{array}$ & $<0.001$ \\
\hline & Moderate & -0.056 & 0.946 & $\begin{array}{l}0.914 \\
0.979\end{array}$ & 0.001 & -0.058 & 0.944 & $\begin{array}{l}0.911 \\
0.978\end{array}$ & 0.001 \\
\hline & Severe & -0.117 & 0.89 & $\begin{array}{l}0.846 \\
0.936\end{array}$ & $<0.001$ & -0.115 & 0.892 & $\begin{array}{l}0.843 \\
0.943\end{array}$ & $<0.001$ \\
\hline & Critical & -0.044 & 0.957 & $\begin{array}{l}0.93 \\
0.985\end{array}$ & 0.003 & -0.036 & 0.964 & $\begin{array}{l}0.938 \\
0.992\end{array}$ & 0.012 \\
\hline
\end{tabular}

prognosis $(P=0.521)$, but this for disease severity had an AUC of 67.45 (cutoff $<257.5 \mathrm{mg} / \mathrm{dL}, P=0.003$ ).

\section{Discussion}

The goal of this study was to explore the iron profile in COVID-19 with the severity of the disease. Fe and transferrin saturation levels were lower in the patient groups, and ferritin levels were higher compared to the controls. In comparison to the control group, $\mathrm{Hb}$ was reduced, whereas $\mathrm{N} / \mathrm{L}$ ratio, ESR, and hs-CRP were elevated. Based on the logistic regression, the increase of ferritin elevated the disease risk, whereas a decline in iron and transferrin saturation reduced the disease risk. Fe was inversely correlated with CRP, ESR, and N/L ratio and these positively correlated with ferritin. $\mathrm{Fe}$, ferritin, and $\mathrm{Hb}$ have higher AUC for disease prognosis, but only ferritin has higher AUC for disease severity assays.

Inflammation is caused by physiological and metabolic changes in the tissue and increase acute phase response includes CRP and ferritin [20]. In response to inflammation, hepcidin transcription is activated by pro-inflammatory cytokines, via the JAK/STAT pathway. Hepcidin triggers the degradation of ferroportin, in enterocytes and macrophages, 
Fig. 3 The ROC curve and the best cutoff for the iron profile and $\mathrm{Hb}$. a Fe ROC curve in patients and critical and severe groups, (a) Fe cutoff in patients, (a) Fe cutoff in critical and severe groups; (b) Ferritin ROC curve in patients and critical and severe groups, (b) ferritin cutoff in patients, (b) ferritin cutoff critical and severe groups; (c) transferrin ROC curve in patients and critical and severe groups, (c) transferrin cutoff in patients, (c) transferrin cutoff in critical and severe groups; (d) Hb ROC curve in patients and critical and severe groups, (d) Hb cutoff in patients, (d) transferrin cutoff in critical and severe groups;

(e) TIBC ROC curve in patients and critical and severe groups, (e) TIBC cutoff in patients, (e) TIBC cutoff in critical and severe groups
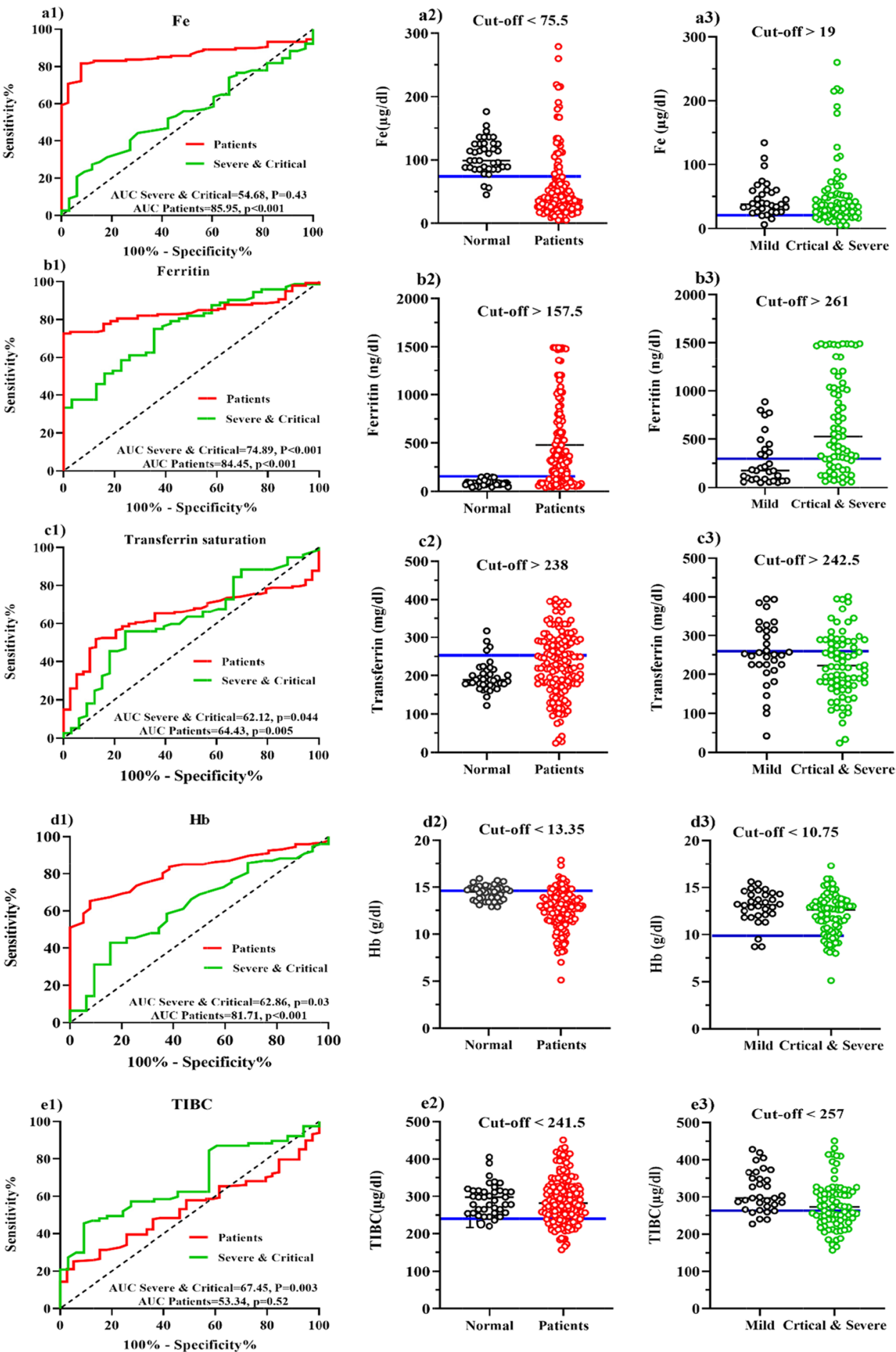

thus blocking iron absorption by negative feedback mechanism. Therefore, iron is retained in macrophages. This causes a rise in systemic inflammation and iron storage protein, i.e., ferritin [21]. Hyperferritinemia not only indicates the acute phase response leading to inflammation but also develop a cytokine storm [22]. Iron, inflammation, and hepcidin in hypoferremia are important elements of the host defense system. Hypoferremia is caused by pro-inflammatory cytokines produced by the host immune cells as a result of inflammation, which increase hepcidin and ferritin expression [23].

Iron is an essential component required for the vital functions of the body and virus $[8,11]$. The innate immune system decreases the transferrin receptor expression in 
macrophages by secreting pro-inflammatory cytokines, and increasing the release of lactoferrin glycoprotein $(80 \mathrm{kDa})$, which functions scavenger of iron [8, 24]. Hepcidin shield the cell against deleterious reactive oxygen species (ROS) such as Fenton, which reduces the absorption of iron and the availability of body fluids [25]. Also, ferritin, whose H-chain exhibits ferroxidase activity, has antioxidant characteristics. It binds to free iron and plays an antioxidant role [26]. So, the immune system reduces oxidative stress by increasing hepcidin and ferritin levels.

Iron chelators have been used to treat cytokine storms and multi-organ injuries in a variety of environments, including ischemia-reperfusion injury, sepsis, and infections. Iron chelation has been shown to help with several viral infections, including HIV-1, hepatitis B, and mengovirus infection, among others [27]. Antiviral and immunomodulatory properties of iron chelator medications might help control SARSCoV-2 and attenuate ARDS through a variety of mechanisms, including the inhibition of viral replication, reduction of iron availability, and up-regulation of B cells [28]. Deferoxamine, deferiprone, and deferasirox are three major FDA-approved iron chelators. Iron chelators bind to the excess iron in the liver and heart muscle cells, leading them to pass through the urine stream. However, in $\mathrm{CoV}$ infections, the mechanism of these drugs has yet to be explored [29]. Anti-IL-6 therapy, such as treatment with ruxolitinib, reduces hepcidin and ferritin levels in COVID-19 patients through the inhibition of the JAK/STAT pathway [30]. Considering the role of IL-6 in the enhancement of the cytokine storm, and the roles of hepcidin and ferritin in inflammation and tissue damage, treatment with IL-6 inhibitor drugs might reduce the cytokine storm by reducing hepcidin and ferritin levels. However, more experiments are needed to prove this hypothesis.

Lower serum iron and higher ferritin levels were associated with decreased lung function in women with asthma [31]. Bolondi et al. [32] reported that iron and transferrin saturation levels dropped in the early days of the illness in COVID-19 patients admitted to the ICU but were restored to normal levels after 7-10 days. After recovery from the disease, due to its vital role in the body, the iron level returns to normal. Hypoferremia and lymphopenia were seen in a study of hypoxemic respiratory failure in COVID-19 by Shah et al. [33], but there was no difference in transferrin saturation and ferritin levels between severe and non-severe patients. Maybe the reason for the lack of difference in transferrin saturation and ferritin levels was the small number of samples (a total of 30 samples, including 10 severe cases and 20 non-severe cases) and that the severe cases admitted to the ICU required invasive mechanical ventilation. We observed lower levels of iron, and N/L ratio in the patients, as well as elevated ESR and hs-CRP levels, indicating infection and inflammation.

Although iron is essential for cell function, it may cause damage to cell proteins, lipids, and DNA by forming hydroxyl free radicals via the Fenton reaction, increasing oxidative stress, and amplifying lipid peroxidation, which can eventually cause ferroptosis and cell death, leading to multi-organ failure [34]. The innate immune system traps iron with the increase in the secretion of hepcidin and ferritin, thereby preventing ferroptosis cell death by free iron.

Hyperferritinemia causes abnormalities in iron homeostasis due to inflammation. Ultimately, inflammation reduces the availability of iron for erythropoiesis, causing anemia, known as anemia of inflammation (AI). This is quite common in people who have infections [35]. We did not observe any symptoms of anemia. $\mathrm{AI}$ is characterized by $\mathrm{Hb}<9.5 \mathrm{~g} /$ $\mathrm{dL}$, transferrin saturation $<16 \%$, and normal or increased ferritin levels (>100 ng/dL). Iron deficiency (ID) anemia is characterized by low iron, transferrin saturation $<16 \%$, and ferritin levels less than $30 \mathrm{ng} / \mathrm{dL}$ [36]. Low Hb levels were observed in male and female COVID-19 patients in the severe and critical groups. Based on the ROC analysis, $\mathrm{Hb}$ values below $13.35 \mathrm{~g} / \mathrm{dL}$ were considered a risk factor. Age of more than 60 years and the male gender have been identified as independent risk factors for death from COVID-19 [37]. In the present study, there was no difference between male and female patients in the study.

Iron and ferritin were shown to be correlated with hospitalization [38]. In a meta-analysis, Henry et al. [39] discovered that ferritin levels in hospitalized patients were significantly higher, which could be a predictor for serious disease development. In a study on mild and severe patients, Bozkurt [40] discovered that an AUC of 88 and a cutoff of $264.5 \mathrm{ng} / \mathrm{mL}$ for ferritin levels could be used to predict disease severity. Cao et al. [41] reported that the cutoff value of $272.5 \mathrm{ng} / \mathrm{mL}$ for ferritin levels (AUC $=87.3$ ) could be used in estimating disease severity upon admission. Ferritin also has a positive correlation with ESR and CRP, because it has pro-inflammatory properties. We also discovered that ferritin cutoff $>261 \mathrm{ng} / \mathrm{dL}$ with an AUC of 74.89 for disease severity in mild vs severe and critical, cutoff $>157.5 \mathrm{ng} / \mathrm{dL}$ with an AUC of 84.45 for disease prediction, and Fe cutoff $<75.5 \mathrm{mg} / \mathrm{dL}$ with an AUC of 85.95 for disease prediction are useful.

The severity of COVID-19 was negatively correlated with serum iron levels while it was positively correlated with CRP [42]. In addition, in our study, ferritin had a positive correlation with $\mathrm{N} / \mathrm{L}$ ratio, ESR, and hs-CRP and a negative correlation with RBC, Hb, HCT, iron, and TIBC. Because ferritin is considered a component of the inflammatory system in infection via protein kinase $\mathrm{C}$, and NF- $\mathrm{KB}$ signaling, developing inflammation induces a drop in Fe and related parameters such as TIBC and RBC, Hb, and HCT [43, 44]. In a study by Lv et al. [45] on patients with severe and non-severe cases of COVID-19, iron, transferrin, and TIBC were decreased and ferritin was increased in severe cases. For serum iron, ferritin, transferrin, the risk ratios were $0.42,4.38$, and 0.19 
respectively. According to our findings, reduction of iron and transferrin saturation had a protective effect, although they enhanced the level of ferritin as a risk factor in patients with COVID-19.

The unique hallmarks of COVID-19 are hyperinflammation and hyperferritinemia. Inflammatory cytokines, particularly IL-6, cause increased hepcidin and ferritin. Ferritin is set by iron availability, inflammation, and hepcidin. As an effect of this iron scavenger in the bloodstream and tissue, iron and ironrelated parameter levels drop dramatically [35]. The strengths of our study were that we divided patients into mild, moderate, severe, and critical and normal groups, and we evaluated iron parameters for the incidence of disease and determined the range for prognosis of disease and disease severity evaluation. Our study limitation is that if the number of samples was more and we had a follow-up and patient mortality and recovery were recorded, by measuring the iron profile, the effect of the iron profile would be more obvious and objective.

\section{Conclusion}

The immune system boosts pro-inflammatory cytokines and expands ferritin and hepcidin production to defend the body by trap iron. The AUC for ferritin and iron for disease prognosis and ferritin for disease severity is higher. Understanding the true link between the iron profile and SARS-CoV-2 infection can help us to minimize the disease symptoms and design treatments based on iron chelators, such as deferoxamine, and decrease the consumption of meals with high iron content.

Supplementary Information The online version contains supplementary material available at https://doi.org/10.1007/s12011-021-03048-8.

Acknowledgements We are grateful for the participants in this study. Also, we express condolences to all those who missed relatives or friends during the coronavirus outbreak.

Author Contribution All authors have accepted responsibility for the entire content of this manuscript and approved its submission.

Funding The work was supported by the Kerman University of Medical Sciences (project no. 99000193).

Data Availability The data described in this study are available upon request.

\section{Declarations}

Ethical Approval This study is approved by the Ethics Committee of Kerman University of Medical Sciences, Kerman, Iran (IR.KMU. REC.1399.239).

Informed Consent Informed consent was obtained from all individuals included in this study.

Competing Interests The authors declare no competing interests.

\section{References}

1. Muniyappa R, Gubbi S (2020) COVID-19 pandemic, coronaviruses, and diabetes mellitus. Am J Physiol Endocrinol Metab 318(5):E736-E741

2. Goudarzi KA et al (2021) Targeted delivery of CRISPR/Cas13 as a promising therapeutic approach to treat SARS-CoV-2. Curr Pharm Biotechnol

3. Poustforoosh A et al (2021) Evaluation of potential anti-RNAdependent RNA polymerase (RdRP) drugs against the newly emerged model of COVID-19 RdRP using computational methods. Biophys Chem 272:106564

4. Taneri PE et al (2020) Anemia and iron metabolism in COVID-19: a systematic review and meta-analysis. medRxiv

5. Ahmadi $\mathrm{Z}$ et al (2019) Association of environmental exposure with hematological and oxidative stress alteration in gasoline station attendants. Environ Sci Pollut Res 26(20):20411-20417

6. Cavezzi A, Troiani E, Corrao S (2020) COVID-19: hemoglobin, iron, and hypoxia beyond inflammation. A narrative review. Clin Pract 10(2)

7. Cassat JE, Skaar EP (2013) Iron in infection and immunity. Cell Host Microbe 13(5):509-519

8. Drakesmith H, Prentice A (2008) Viral infection and iron metabolism. Nat Rev Microbiol 6(7):541-552

9. Northrop-Clewes CA (2008) Interpreting indicators of iron status during an acute phase response-lessons from malaria and human immunodeficiency virus. Ann Clin Biochem 45(1):18-32

10. Yamanishi $\mathrm{H}$ et al (2002) Modification of fully automated total iron-binding capacity (TIBC) assay in serum and comparison with dimension TIBC method. Clin Chem 48(9):1565-1570

11. Boyd PW et al (2007) Mesoscale iron enrichment experiments 1993-2005: synthesis and future directions. Science 315(5812):612-617

12. Tarifeño-Saldivia $E$ et al (2018) Iron overload is associated with oxidative stress and nutritional immunity during viral infection in fish. Front Immunol 9:1296

13. Sebastiani G, Tempesta D, Alberti A (2012) Hepatic iron overload is common in chronic hepatitis B and is more severe in patients coinfected with hepatitis D virus. J Viral Hepatitis 19(2):e170-e176

14. Khan FA, Fisher MA, Khakoo RA (2007) Association of hemochromatosis with infectious diseases: expanding spectrum. Int $\mathrm{J}$ Infect Dis 11(6):482-487

15 Fillebeen C, Pantopoulos K (2010) Iron inhibits replication of infectious hepatitis $\mathrm{C}$ virus in permissive Huh7. 5.1 cells. J Hepatol 53(6):995-999

16. Gonçalves JL et al (2019) Iron intake is positively associated with viral load in antiretroviral naïve Brazilian men living with HIV. Memórias do Instituto Oswaldo Cruz, 114

17. Alipour R, Hashemi SH, Mikaeili F (2020) Serum iron level in patients with COVID-19: a case report study. Int J Res Med Sci $8(7): 2658$

18. Gandhi RT, Lynch JB, Del Rio C (2020) Mild or moderate COVID-19. N Engl J Med 383(18):1757-1766

19. Asadikaram $\mathrm{G}$ et al (2019) The study of the serum level of IL- 4 , TGF- $\beta$, IFN- $\gamma$, and IL- 6 in overweight patients with and without diabetes mellitus and hypertension. J Cell Biochem 120(3):4147-4157

20. Lagadinou $\mathrm{M}$ et al (2020) Prognosis of COVID-19: Changes in laboratory parameters. Age (yrs) 62(13.4):47-16.42

21. Edeas M, Saleh J, Peyssonnaux C (2020) Iron: innocent bystander or vicious culprit in COVID-19 pathogenesis? Int J Infect Dis 97:303-305 
22. Rosário C et al (2013) The hyperferritinemic syndrome: macrophage activation syndrome, Still's disease, septic shock and catastrophic antiphospholipid syndrome. BMC Med 11(1):1-11

23. Fleming RE (2008) Iron and inflammation: cross-talk between pathways regulating hepcidin. J Mol Med 86(5):491-494

24. Nairz $M$ et al (2010) The struggle for iron-a metal at the hostpathogen interface. Cell Microbiol 12(12):1691-1702

25. Zhang Q, Huang X (2003) Induction of interleukin-6 by coal containing bioavailable iron is through both hydroxyl radical and ferryl species. J Biosci 28(1):95-100

26 Arosio P, Levi S (2010) Cytosolic and mitochondrial ferritins in the regulation of cellular iron homeostasis and oxidative damage. Biochim Biophys Acta BBA Gen Subj 1800(8):783-792

27. Vlahakos VD et al (2020) Hyperferritinemia in patients with COVID-19: an opportunity for iron chelation? Artif Organs

28. Dalamaga M, Karampela I, Mantzoros CS (2020) Commentary: could iron chelators prove to be useful as an adjunct to COVID-19 Treatment Regimens? Metabolism 108:154260

29. Abbas A et al (2020) Use of iron chelators to reduce the severity of COVID-19. Thromb Haemost Res 4(2):1042

30. Innes AJ et al (2020) Ruxolitinib for tocilizumab-refractory severe COVID-19 infection. Br J Haematol 190(4):e198-e200

31. Brigham EP et al (2015) Iron status is associated with asthma and lung function in US women. PloS One 10(2):e0117545

32. Bolondi $\mathrm{G}$ et al (2020) Iron metabolism and lymphocyte characterisation during COVID-19 infection in ICU patients: an observational cohort study. World J Emerg Surg 15(1):1-8

33. Shah A et al (2020) Systemic hypoferremia and severity of hypoxemic respiratory failure in COVID-19. Crit Care 24(1):1-4

34. Doll S, Conrad M (2017) Iron and ferroptosis: a still ill-defined liaison. IUBMB Life 69(6):423-434

35. Sonnweber $\mathrm{T}$ et al (2020) Persisting alterations of iron homeostasis in COVID-19 are associated with non-resolving lung pathologies and poor patients' performance: a prospective observational cohort study. Respir Res 21(1):1-9

36 Bergamaschi G et al (2010) Prevalence and pathogenesis of anemia in inflammatory bowel disease. Influence of anti-tumor necrosis factor- $\alpha$ treatment. Haematologica 95(2):199
37. Wang D et al (2020) Clinical course and outcome of 107 patients infected with the novel coronavirus, SARS-CoV-2, discharged from two hospitals in Wuhan. China Critical Care 24:1-9

38. Hippchen T et al (2020) Hypoferremia predicts hospitalization and oxygen demand in COVID-19 patients. Available at SSRN 3608074

39. Henry BM et al (2020) Hematologic, biochemical and immune biomarker abnormalities associated with severe illness and mortality in coronavirus disease 2019 (COVID-19): a meta-analysis. Clin Chem Lab Med 58(7):1021-1028

40. Bozkurt FT et al (2021) Can ferritin levels predict the severity of illness in patients with COVID-19? Cureus 13(1)

41. Cao P et al (2021) Elevated serum ferritin level effectively discriminates severity illness and liver injury of coronavirus disease 2019 pneumonia. Biomarkers 26(3):207-212

42. Zhao K et al (2020) Serum iron level as a potential predictor of coronavirus disease 2019 severity and mortality: a retrospective study. in Open forum infectious diseases. Oxford University Press US

43. Peng YY, Uprichard J (2017) Ferritin and iron studies in anaemia and chronic disease. Ann Clin Biochem 54(1):43-48

44. Ruddell RG et al (2009) Ferritin functions as a proinflammatory cytokine via iron-independent protein kinase $\mathrm{C}$ zeta/nuclear factor kappaB-regulated signaling in rat hepatic stellate cells. Hepatology 49(3):887-900

45. Lv Y et al (2021) Association between iron status and the risk of adverse outcomes in COVID-19. Clin Nutr 40(5):3462-3469

Publisher's Note Springer Nature remains neutral with regard to jurisdictional claims in published maps and institutional affiliations. 